\title{
Generalized fractional integral operators and the multivariable $H$-function
}

\author{
Praveen Agarwal ${ }^{1}$, Sergei V Rogosin'², Erkinjon T Karimov ${ }^{3 *}$ and Mehar Chand ${ }^{4}$
}

"Correspondence:

erkinjon@gmail.com

${ }^{3}$ Institute of Mathematics, National University of Uzbekistan, Tashkent, 100125, Uzbekistan

Full list of author information is

available at the end of the article

\begin{abstract}
The main object of the present paper is to establish new fractional integral formulas (of Marichev-Saigo-Maeda type) involving the products of the multivariable $\mathrm{H}$-functions and the first class of multivariable polynomials due to Srivastava and Garg. All the results derived here are of general character and can yield a number of (new and known) results in the theory of fractional calculus.
\end{abstract}

MSC: 26A33; 33C45; 33C60; 33C70; 33E12

Keywords: Marichev-Saigo-Maeda fractional integral operator; multivariable $H$-function; first class of multivariable polynomials; Mittag-Leffler function

\section{Introduction}

Nowadays, fractional calculus is used as a mathematical tool for modeling the processes in engineering, mathematical economics, physics, chemistry, biology and other branches of science (see [1-12]). Motivated by the above mentioned works, here we aim to establish two new fractional integral formulas (of Marichev-Saigo-Maeda type) involving the product of multivariable $H$-functions and the first class of multivariable polynomials $S_{n}^{m_{1}, \ldots, m_{s}}\left(x_{1}, \ldots, x_{s}\right)$. The so-called Marichev-Saigo-Maeda generalized fractional operators were introduced nearly 40 years ago by Marichev [13] and studied in some recent papers, including the papers by Saigo and Maeda [14] and by Saxena and Saigo [15]. Due to the usefulness and the importance of the Marichev-Saigo-Maeda fractional integral operators, many authors have presented a number of interesting integral formulas involving special functions by using the Marichev-Saigo-Maeda fractional integral operator (see $[13,16-20])$. By virtue of the unified nature of Marichev-Saigo-Maeda fractional integral operators, a large number of new and known results involving Saigo, Riemann-Liouville and Erdélyi-Kober fractional integral operators follow as special cases of our main formulas.

Our main results consist in application of the Marichev-Saigo-Maeda generalized fractional operators:

$$
\begin{aligned}
& \left(\mathrm{I}_{0, x}^{\alpha, \alpha^{\prime}, \beta, \beta^{\prime}, \eta} f\right)(x) \\
& \quad=\frac{x^{-\alpha}}{\Gamma(\eta)} \int_{0}^{x}(x-t)^{\eta-1} t^{-\alpha^{\prime}} F_{3}\left(\alpha, \alpha^{\prime}, \beta, \beta^{\prime} ; \eta ; 1-\frac{t}{x}, 1-\frac{x}{t}\right) f(t) d t \quad(\operatorname{Re} \eta>0)
\end{aligned}
$$

(c) 2015 Agarwal et al. This article is distributed under the terms of the Creative Commons Attribution 4.0 International License (http://creativecommons.org/licenses/by/4.0/), which permits unrestricted use, distribution, and reproduction in any medium, provided you give appropriate credit to the original author(s) and the source, provide a link to the Creative Commons license, and indicate if changes were made. 
and

$$
\begin{aligned}
& \left(\mathrm{I}_{x, \infty}^{\alpha, \alpha^{\prime}, \beta, \beta^{\prime}, \eta} f\right)(x) \\
& \quad=\frac{x^{-\alpha^{\prime}}}{\Gamma(\eta)} \int_{x}^{\infty}(t-x)^{\eta-1} t^{-\alpha} F_{3}\left(\alpha, \alpha^{\prime}, \beta, \beta^{\prime} ; \eta ; 1-\frac{x}{t}, 1-\frac{t}{x}\right) f(t) d t \quad(\operatorname{Re} \eta>0),
\end{aligned}
$$

to the multivariable $H$-functions (see, e.g., $[21,22]$ ) and the first class of multivariable polynomials $S_{n}^{m_{1}, \ldots, m_{s}}\left(x_{1}, \ldots, x_{s}\right)$ given by $(1.15)$.

A variant of such operators (integral transforms) was introduced by Marichev [13] as Mellin type convolution operators with a special function $F_{3}(\cdot)$ in the kernel. These operators were rediscovered and studied by Saigo in [23] as a generalization of the so-called Saigo fractional integral operators, see [24]. The properties of these operators were studied by Saigo and Maeda [14]. In particular, relations of the operators with the Mellin transforms, hypergeometric operators (or Saigo fractional integral operators), their decompositions and acting properties in the McBride spaces $F_{p ; \mu}$ (see [25]) were found.

In (1.1), (1.2) the symbol $F_{3}(\cdot)$ denotes the so-called 3rd Appell function (known also as Horn function) (see [26], p.413):

$$
F_{3}\left(\alpha, \alpha^{\prime} ; \beta, \beta^{\prime} ; \eta ; x ; y\right)=\sum_{m, n=0}^{\infty} \frac{(\alpha)_{m}\left(\alpha^{\prime}\right)_{n}(\beta)_{m}\left(\beta^{\prime}\right)_{n}}{(\eta)_{m+n} m ! n !} x^{m} y^{n} \quad(\max \{|x|,|y|\}<1) .
$$

The properties of this function are discussed in [26], pp.412-415. In particular, its relation to the Gauss hypergeometric function is presented:

$$
F_{3}(\alpha, \eta-\alpha ; \beta, \eta-\beta ; \eta ; x ; y)={ }_{2} F_{1}(\alpha, \beta ; \eta ; x+y-x y) .
$$

It is known that the $3 \mathrm{rd}$ Appell function cannot be expressed as a product of two ${ }_{2} F_{1}$ functions and satisfy pairs of linear partial differential equations of the second order.

Operators (1.1), (1.2) can be reduced to the fractional integral operators introduced by Saigo [27] due to the following relations:

$$
\mathrm{I}_{0, x}^{\alpha, 0, \beta, \beta^{\prime}, \gamma} f(x)=\mathrm{I}_{0, x}^{\gamma, \alpha-\gamma,-\beta} f(x) \quad(\gamma \in \mathbb{C})
$$

and

$$
\mathrm{I}_{x, \infty}^{\alpha, 0, \beta, \beta^{\prime}, \gamma} f(x)=\mathrm{I}_{x, \infty}^{\gamma, \alpha-\gamma,-\beta} f(x) \quad(\gamma \in \mathbb{C}) .
$$

The multivariable $H$-function, introduced by Srivastava and Panda in a series of research papers (see [28], p.271, Eq. (4.1), [22], p.130, Eq. $(1.3)$ and [29, 30]), can be defined and represented in the following manner (see, e.g., [31], p.251, Eq. (C.1)): ${ }^{\mathrm{a}}$

$$
\begin{aligned}
H\left[x_{1}, \ldots, x_{r}\right] & =H_{p, q:\left\{p_{r}, q_{r}\right\}}^{0,\left\{m_{r}, n_{r}\right\}}\left[\begin{array}{c}
x_{1} \\
\vdots \\
x_{r}
\end{array} \mid \begin{array}{l}
\left.\left(a_{j} ; \alpha_{j}^{(1)}, \ldots, \alpha_{j}^{(r)}\right)_{1, p}:\left\{\left(c_{j}^{(r)}, \gamma_{j}^{(r)}\right)_{\left.1, p_{r}\right\}}\right\}, \ldots, \beta_{j}^{(r)}\right)_{1, q}:\left\{\left(d_{j}^{(r)}, \delta_{j}^{(r)}\right)_{1, q_{r}}\right\} \\
\left(b_{j}\right)
\end{array}\right. \\
& =\frac{1}{(2 \pi \omega)^{r}} \int_{L_{1}} \ldots \int_{L_{r}} \phi\left(\xi_{1}, \ldots, \xi_{r}\right) \prod_{i=1}^{r}\left(\theta_{i}\left(\xi_{i}\right) x_{i}^{\xi_{i}} d \xi_{i}\right),
\end{aligned}
$$


where

$$
\begin{gathered}
\phi\left(\xi_{1}, \ldots, \xi_{r}\right)=\frac{\prod_{j=1}^{n} \Gamma\left(1-a_{j}+\sum_{i=1}^{r} \alpha_{j}^{(i)} \xi_{i}\right)}{\prod_{j=n+1}^{p} \Gamma\left(a_{j}-\sum_{i=1}^{r} \alpha_{j}^{(i)} \xi_{i}\right) \prod_{j=1}^{q} \Gamma\left(1-b_{j}+\sum_{i=1}^{r} \beta_{j}^{(i)} \xi_{i}\right)}, \\
\theta_{i}\left(\xi_{i}\right)=\frac{\prod_{j=1}^{n_{i}} \Gamma\left(1-c_{j}^{(i)}+\gamma_{j}^{(i)} \xi_{i}\right) \prod_{j=1}^{m_{i}} \Gamma\left(d_{j}^{(i)}-\delta_{j}^{(i)} \xi_{i}\right)}{\prod_{j=n_{i}+1}^{p_{i}} \Gamma\left(c_{j}^{(i)}-\gamma_{j}^{(i)} \xi_{i}\right) \prod_{j=m_{i}+1}^{q_{i}} \Gamma\left(1-d_{j}^{(i)}+\delta_{j}^{(i)} \xi_{i}\right)}, \quad \forall i \in\{1,2, \ldots, r\} .
\end{gathered}
$$

In these formulas, for any $i=1, \ldots, r, L_{i}$ represents the contours which start at the point $\tau_{i}-\omega \infty$ and go $\tau_{i}+\omega \infty$ with $\tau_{i} \in \mathbb{R}=(-\infty, \infty)$ such that all the poles of $\Gamma\left(d_{j}^{(i)}-\delta_{j}^{(i)} \xi_{i}\right)$, $j=1, \ldots, m_{i} ; i=1, \ldots, r$, are separated from those of $\Gamma\left(1-c_{j}^{(i)}+\gamma_{j}^{(i)} \xi_{i}\right), j=1, \ldots, n_{i} ; i=1, \ldots, r$, and $\Gamma\left(1-a_{j}+\sum_{i=1}^{r} \alpha_{j}^{(i)} \xi_{i}\right), j=1, \ldots, n$. Here, the integers $n, p, q, m_{i}, n_{i}, p_{i}, q_{i}$ satisfy the inequalities $0 \leq n \leq p ; q \geq 0,1 \leq m_{i} \leq q_{i}$ and $1 \leq n_{i} \leq p_{i}, i=1, \ldots, r$.

Further, suppose that the parameters $a_{j}, j=1, \ldots, p ; c_{j}^{(i)}, j=1, \ldots, p_{i} ; i=1, \ldots, r, b_{j}, j=$ $1, \ldots, q ; d_{j}^{(i)}, j=1, \ldots, q_{i} ; i=1, \ldots, r$, are complex numbers, while the associated coefficients $\alpha_{j}^{(i)}, j=1, \ldots, p ; i=1, \ldots, r ; \gamma_{j}^{(i)}, j=1, \ldots, p_{i} ; i=1, \ldots, r, \beta_{j}^{(i)}, j=1, \ldots, q ; i=1, \ldots, r ; \delta_{j}^{(i)}, j=$ $1, \ldots, q_{i} ; i=1, \ldots, r$, are positive real numbers such that

$$
\begin{aligned}
& \Lambda_{i}=\sum_{j=1}^{p} \alpha_{j}^{(i)}+\sum_{j=1}^{p_{i}} \gamma_{j}^{(i)}-\sum_{j=1}^{q} \beta_{j}^{(i)}-\sum_{j=1}^{q_{i}} \delta_{j}^{(i)} \leq 0, \\
& \Omega_{i}=\sum_{j=1}^{n} \alpha_{j}^{(i)}-\sum_{j=n+1}^{p} \alpha_{j}^{(i)}+\sum_{j=1}^{q} \beta_{j}^{(i)}+\sum_{j=1}^{n_{i}} \gamma_{j}^{(i)}-\sum_{j=n_{i}+1}^{p_{i}} \gamma_{j}^{(i)}+\sum_{j=1}^{m_{i}} \delta_{j}^{(i)}-\sum_{j=m_{i}+1}^{q_{i}} \delta_{j}^{(i)}>0 .
\end{aligned}
$$

It is assumed that the poles of integrand of (1.6) are simple. Under the conditions (1.10) the integrals in (1.6) converge absolutely (see [31], p.251) in the domain

$$
\left|\arg \left(z_{i}\right)\right|<\frac{\pi}{2} \Omega_{i}, \quad i=1, \ldots, r,
$$

and points $z_{i}=0, i=1, \ldots, r$, and various exceptional parameter values being tacitly excluded.

From Srivastava and Panda [32], we have

$$
H\left[z_{1}, \ldots, z_{r}\right]=o\left(\left|z_{1}\right|^{e_{1}}, \ldots,\left|z_{r}\right|^{e_{r}}\right), \quad \max _{1 \leq j \leq r}\left[\left|z_{j}\right|\right] \rightarrow 0,
$$

where $e_{i}=\min _{1 \leq j \leq m_{i}}\left[\frac{\Re\left(d_{j}^{(i)}\right)}{\delta_{j}^{(i)}}\right], i=1, \ldots, r$.

If $n=0$ in (1.6), then the following asymptotic expansion (Srivastava and Panda [32]) holds:

$$
H\left[z_{1}, \ldots, z_{r}\right]=o\left(\left|z_{1}\right|^{g_{1}}, \ldots,\left|z_{r}\right|^{g_{r}}\right), \quad \min _{1 \leq j \leq r}\left[\left|z_{j}\right|\right] \rightarrow \infty
$$

where $g_{i}=\max _{1 \leq j \leq m_{i}}\left[\frac{\Re\left(c_{j}^{(i)}\right)}{\gamma_{j}^{(i)}}\right], i=1, \ldots, r$, provided that (1.9), (1.10) and (1.11) hold true.

Remark 1 When $n=2$, the multivariable $H$-function defined by (1.6) reduces to the $H$-function of two variables studied by Mittal and Gupta [33]. The $H$-function of two 
variables is also defined and studied by Munot and Kalla [34], Verma [35] and Hai and Yakubovich [36].

Remark 2 It is interesting to observe that for $n=p=q=0$, the multivariable $H$-function breaks up into the product of $r, H$-functions; and consequently, there holds the following result (Saxena [21]):

$$
H_{0,0:\left\{p_{r}, q_{r}\right\}}^{0,0:\left\{m_{r}\right\}}\left[\begin{array}{c|c}
x_{1} & -:\left\{\left(c_{j}^{(r)}, \gamma_{j}^{(r)}\right)_{1, p_{r}}\right\} \\
\vdots & -:\left\{\left(d_{j}^{(r)}, \delta_{j}^{(r)}\right)_{1, q_{r}}\right\} \\
x_{r}
\end{array}\right]=\prod_{i=1}^{r} H_{p_{i}, q_{i}}^{m_{i}, n_{i}}\left[z_{i} \mid \begin{array}{l}
\left\{\left(c_{j}^{(i)}, \gamma_{j}^{(i)}\right)_{1, p_{i}}\right\} \\
\left\{\left(d_{j}^{(i)}, \delta_{j}^{(i)}\right)_{1, q_{i}}\right\}
\end{array}\right] .
$$

Remark 3 The function defined by (1.6) was introduced and studied by Srivastava and Panda [37]. When $\alpha_{j}^{(1)}=\cdots=\alpha_{j}^{(r)}, j=1, \ldots, p ; \beta_{j}^{(1)}=\cdots=\beta_{j}^{(r)}, j=1, \ldots, q$, in (1.6) the multivariable $H$-function defined and studied by Saxena [21,38] is obtained. In case all the Greek letters are assumed to be unity, the $H$-function of several complex variables (1.6) reduces to the G-function of several complex variables studied by Khadia and Goyal [39, 40].

Remark 4 Fractional integrals involving multivariable $H$-functions are given in a series of papers by Saigo and Saxena [41-43], Srivastava and Hussain [44], Saigo et al. [45] and others.

In the sequel, Srivastava and Garg [46], p.686, Eq. (1.4), introduced the multivariable analogue of the polynomials $S_{n}^{m}(x)$. This first class of multivariable polynomials $S_{n}^{m_{1}, \ldots, m_{s}}\left(x_{1}, \ldots, x_{s}\right)$ is defined by

$$
S_{n}^{m_{1}, \ldots, m_{s}}\left(x_{1}, \ldots, x_{s}\right)=\sum_{k_{1}, \ldots, k_{s}=0}^{m_{1} k_{1}+\cdots+m_{s} k_{s} \leq n}(-n)_{m_{1} k_{1}+\cdots+m_{s} k_{s}} \Lambda\left(n ; k_{1}, \ldots, k_{s}\right) \frac{x_{1}^{k_{1}} \cdots x_{s}^{k_{s}}}{k_{1} ! \cdots k_{s} !},
$$

where $m_{1}, \ldots, m_{s}$ are arbitrary positive integers and the coefficients $\Lambda\left(n ; k_{1}, \ldots, k_{s}\right)\left(n, k_{i} \geq\right.$ $0, i=1, \ldots, s)$ are arbitrary constants, real or complex.

\section{Main results}

In this section, we prove two theorems on composition of the Marichev-Saigo-Maeda operators with the product of a multivariable $H$-function and the first class of multivariable polynomials. We start with presenting image formulas involving Marichev-Saigo-Maeda fractional integral operators.

Lemma 1 (see [14]) Let $\alpha, \alpha^{\prime}, \beta, \beta^{\prime}, \gamma \in \mathbb{C}$.

$$
\begin{aligned}
& \text { If } \Re(\gamma)>0, \Re(p)>\max \left[0, \Re\left(\alpha+\alpha^{\prime}+\beta-\gamma\right), \Re\left(\alpha^{\prime}-\beta^{\prime}\right)\right] \text {, then } \\
& \mathrm{I}_{0, x}^{\alpha, \alpha^{\prime}, \beta, \beta^{\prime}, \gamma} x^{\rho-1}=x^{\rho-\alpha-\alpha^{\prime}+\gamma-1} \frac{\Gamma(\rho) \Gamma\left(\rho+\gamma-\alpha-\alpha^{\prime}-\beta\right) \Gamma\left(\rho+\beta^{\prime}-\alpha^{\prime}\right)}{\Gamma\left(\rho+\gamma-\alpha-\alpha^{\prime}\right) \Gamma\left(\rho+\gamma-\alpha^{\prime}-\beta\right) \Gamma\left(\rho+\beta^{\prime}\right)} . \\
& \text { If } \Re(\gamma)>0, \Re(p)<1+\min \left[\Re(-\beta), \Re\left(\alpha+\alpha^{\prime}-\gamma\right), \Re\left(\alpha+\beta^{\prime}-\gamma\right)\right] \text {, then } \\
& \mathrm{I}_{x, \infty}^{\alpha, \alpha^{\prime}, \beta, \beta^{\prime}, \gamma} x^{\rho-1}=x^{\rho-\alpha-\alpha^{\prime}+\gamma-1} \\
& \times \frac{\Gamma\left(1+\alpha+\alpha^{\prime}-\gamma-\rho\right) \Gamma\left(1+\alpha+\beta^{\prime}-\gamma-\rho\right) \Gamma(1-\beta-\rho)}{\Gamma(1-\rho) \Gamma\left(1+\alpha+\alpha^{\prime}+\beta^{\prime}-\gamma-\rho\right) \Gamma(1+\alpha-\beta-\rho)} .
\end{aligned}
$$


Theorem 1 Let $\Re(\gamma)>0$ and $\alpha, \alpha^{\prime}, \beta, \beta^{\prime}, \gamma, \mu, \eta, \delta_{j}, v_{i}, z_{i}, a, b, c_{j} \in \mathbb{C}, \lambda_{j}, \sigma_{i}>0(i \in\{1, \ldots, r\}$; $j \in\{1, \ldots, s\})$. Let the following conditions be satisfied, too:

(i) $\left|\arg z_{i}\right|<\frac{\pi}{2} \Omega_{i}, \Omega_{i}>0(i=1, \ldots, r)$, where

$$
\begin{aligned}
\Omega_{i}= & \sum_{j=1}^{n} \alpha_{j}^{(i)}-\sum_{j=n+1}^{p} \alpha_{j}^{(i)}-\sum_{j=1}^{q} \beta_{j}^{(i)}+\sum_{j=1}^{n_{i}} \gamma_{j}^{(i)} \\
& -\sum_{j=n_{i}+1}^{p_{i}} \gamma_{j}^{(i)}+\sum_{j=1}^{m_{i}} \delta_{j}^{(i)}-\sum_{j=m_{i}+1}^{q_{i}} \delta_{j}^{(i)}>0 .
\end{aligned}
$$

(ii) $\min _{1 \leq j \leq m_{i}, 1 \leq i \leq r}\left[-\frac{\sigma_{i} \Re\left(d_{j}^{(i)}\right)}{\delta_{j}^{(i)}}\right]<\Re(\mu)+\min \left[\Re\left(\alpha^{\prime}\right), \Re\left(\beta^{\prime}\right), \Re(\gamma-\alpha-\beta)\right]$.

(iii) $\left|\frac{a}{b} x\right|<1$, also we have

$$
\begin{aligned}
& \Re(\mu)+\sum_{i=1}^{r} \sigma_{i} \min _{1 \leq j \leq m_{i}}\left[\frac{\Re\left(d_{j}^{(i)}\right)}{\delta_{j}^{(i)}}\right] \\
& >\max \left[0, \Re\left(\alpha+\alpha^{\prime}+\beta-\gamma\right), \Re\left(\alpha^{\prime}-\beta^{\prime}\right)\right] \quad(i=1, \ldots, r), \\
& \Re(\eta)+\sum_{i=1}^{r} v_{i} \min _{1 \leq j \leq m_{i}}\left[\frac{\Re\left(d_{j}^{(i)}\right)}{\delta_{j}^{(i)}}\right] \\
& >\max \left[0, \Re\left(\alpha+\alpha^{\prime}+\beta-\gamma\right), \Re\left(\alpha^{\prime}-\beta^{\prime}\right)\right] \quad(i=1, \ldots, r) .
\end{aligned}
$$

Then the following result holds:

$$
\begin{aligned}
& \left\{\mathrm { I } _ { 0 + } ^ { \alpha , \alpha ^ { \prime } , \beta , \beta ^ { \prime } , \gamma } \left(t^{\mu-1}(b-a t)^{-\eta} S_{n}^{m_{1}, \ldots, m_{s}}\left[c_{1} t^{\lambda_{1}}(b-a t)^{-\delta_{1}}, \ldots, c_{s} t^{\lambda_{s}}(b-a t)^{-\delta_{s}}\right]\right.\right. \\
& \left.\left.\times H\left[z_{1} t^{\sigma_{1}}(b-a t)^{-v_{1}}, \ldots, z_{r} t^{\sigma_{r}}(b-a t)^{-v_{r}}\right]\right)\right\}(x) \\
& =b^{-\eta} x^{\mu-\alpha-\alpha^{\prime}+\gamma-1} \sum_{k_{1}, \ldots, k_{s}=0}^{m_{1} k_{1}+\cdots+m_{s} k_{s} \leq n} \frac{(-n)_{m_{1} k_{1}+\cdots+m_{s} k_{s}}}{k_{1} ! \cdots k_{s} !} \Lambda\left(n ; k_{1}, \ldots, k_{s}\right) c_{1}^{k_{1}} \cdots c_{s}^{k_{s}} \\
& \times b^{-\sum_{j=1}^{s} \delta_{j} k_{j}} x^{\sum_{j=1}^{s} \lambda_{j} k_{j}} H_{p+4, q+4:\left\{p_{r}, q r\right\} ; 0,1}^{0, n+4:\left\{m_{r}, n_{n}\right\}: 1,0}
\end{aligned}
$$

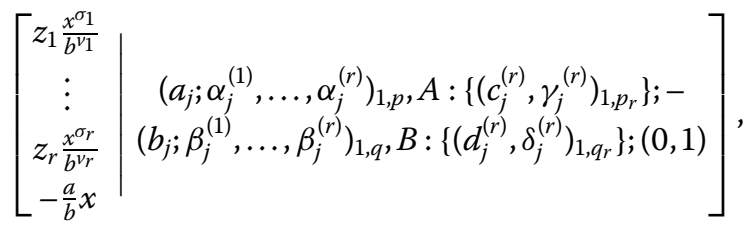

where

$$
\begin{aligned}
A= & \left(1-\eta-\sum_{j=1}^{s} \delta_{j} k_{j} ; v_{1}, \ldots, v_{r}, 1\right),\left(1-\mu-\sum_{j=1}^{s} \lambda_{j} k_{j} ; \sigma_{1}, \ldots, \sigma_{r}, 1\right), \\
& \left(1-\mu-\gamma+\alpha+\alpha^{\prime}+\beta-\sum_{j=1}^{s} \lambda_{j} k_{j} ; \sigma_{1}, \ldots, \sigma_{r}, 1\right) \\
& \left(1-\mu+\alpha^{\prime}-\beta^{\prime}-\sum_{j=1}^{s} \lambda_{j} k_{j} ; \sigma_{1}, \ldots, \sigma_{r}, 1\right)
\end{aligned}
$$


and

$$
\begin{aligned}
B= & \left(1-\eta-\sum_{j=1}^{s} \delta_{j} k_{j} ; \nu_{1}, \ldots, v_{r}, 0\right),\left(1-\mu-\gamma+\alpha+\alpha^{\prime}-\sum_{j=1}^{s} \lambda_{j} k_{j} ; \sigma_{1}, \ldots, \sigma_{r}, 1\right) \\
& \left(1-\mu-\gamma+\alpha^{\prime}+\beta-\sum_{j=1}^{s} \lambda_{j} k_{j} ; \sigma_{1}, \ldots, \sigma_{r}, 1\right), \\
& \left(1-\mu-\beta^{\prime}-\sum_{j=1}^{s} \lambda_{j} k_{j} ; \sigma_{1}, \ldots, \sigma_{r}, 1\right) .
\end{aligned}
$$

Theorem 2 Let $\alpha, \alpha^{\prime}, \beta, \beta^{\prime}, \gamma, \mu, \eta, \delta_{j}, v_{i}, z_{i}, a, b, c_{j} \in \mathbb{C}, \lambda_{j}, \sigma_{i}>0(i \in\{1, \ldots, r\} ; j \in\{1, \ldots, s\})$ and $\mathfrak{R}(\gamma)>0$. Let the following conditions be satisfied, too:

$$
\begin{aligned}
& \Re(\mu)-\sum_{i=1}^{r} \sigma_{i} \min _{1 \leq j \leq m_{i}}\left[\frac{\Re\left(d_{j}^{(i)}\right)}{\delta_{j}^{(i)}}\right]<1+\max \left[\Re(-\beta), \Re\left(\alpha+\alpha^{\prime}-\gamma\right), \Re\left(\alpha+\beta^{\prime}-\gamma\right)\right], \\
& \Re(\eta)-\sum_{i=1}^{r} v_{i} \min _{1 \leq j \leq m_{i}}\left[\frac{\Re\left(d_{j}^{(i)}\right)}{\delta_{j}^{(i)}}\right]<1+\max \left[\Re(-\beta), \Re\left(\alpha+\alpha^{\prime}-\gamma\right), \Re\left(\alpha+\beta^{\prime}-\gamma\right)\right],
\end{aligned}
$$

and the conditions (i)-(iii) in Theorem 1 are also satisfied.

Then the following result holds:

$$
\begin{aligned}
& \left\{\mathrm { I } _ { 0 - } ^ { \alpha , \alpha ^ { \prime } , \beta , \beta ^ { \prime } , \gamma } \left(t^{\mu-1}(b-a t)^{-\eta} S_{n}^{m_{1}, \ldots, m_{s}}\left[c_{1} t^{\lambda_{1}}(b-a t)^{-\delta_{1}}, \ldots, c_{s} t^{\lambda_{s}}(b-a t)^{-\delta_{s}}\right]\right.\right. \\
& \left.\left.\times H\left[z_{1} t^{\sigma_{1}}(b-a t)^{-v_{1}}, \ldots, z_{r} t^{\sigma_{r}}(b-a t)^{-v r}\right]\right)\right\}(x) \\
& =b^{-\eta} x^{\mu-\alpha-\alpha^{\prime}+\gamma-1} \sum_{k_{1}, \ldots, k_{s}=0}^{m_{1} k_{1}+\cdots+m_{s} k_{s} \leq n} \frac{(-n)_{m_{1} k_{1}+\cdots+m_{s} k_{s}}}{k_{1} ! \cdots k_{s} !} \Lambda\left(n ; k_{1}, \ldots, k_{s}\right) c_{1}^{k_{1}} \cdots c_{s}^{k_{s}} \\
& \times b^{-\sum_{j=1}^{s} \delta_{j} k_{j}} x^{\sum_{j=1}^{s} \lambda_{j} k_{j}} H_{p+4, q+4:\left\{p_{r}, q_{r}\right\} ; 0,1}^{0, n+4:\left\{m_{r}, n_{r}\right\} ; 1,0} \\
& {\left[\begin{array}{c|c}
z_{1} \frac{x^{\sigma_{1}}}{b^{v_{1}}} & \left(a_{j} ; \alpha_{j}^{(1)}, \ldots, \alpha_{j}^{(r)}\right)_{1, p}, C:\left\{\left(c_{j}^{(r)}, \gamma_{j}^{(r)}\right)_{1, p_{r}}\right\} ;- \\
\vdots & \left(b_{j} ; \beta_{j}^{(1)}, \ldots, \beta_{j}^{(r)}\right)_{1, q}, D:\left\{\left(d_{j}^{(r)}, \delta_{j}^{(r)}\right)_{1, q_{r}}\right\} ;(0,1) \\
z_{r} \frac{x^{\sigma_{r}}}{b^{v_{r}}} & \\
-\frac{a}{b} x &
\end{array}\right],}
\end{aligned}
$$

where

$$
\begin{aligned}
C= & \left(1-\eta-\sum_{j=1}^{s} \delta_{j} k_{j} ; v_{1}, \ldots, v_{r}, 1\right),\left(1-\mu-\sum_{j=1}^{s} \lambda_{j} k_{j} ; \sigma_{1}, \ldots, \sigma_{r}, 1\right) \\
& \left(1+\alpha+\alpha^{\prime}+\beta^{\prime}-\mu-\gamma-\sum_{j=1}^{s} \lambda_{j} k_{j} ; \sigma_{1}, \ldots, \sigma_{r}, 1\right), \\
& \left(1+\alpha-\beta-\mu-\sum_{j=1}^{s} \lambda_{j} k_{j} ; \sigma_{1}, \ldots, \sigma_{r}, 1\right)
\end{aligned}
$$


and

$$
\begin{aligned}
D= & \left(1-\eta-\sum_{j=1}^{s} \delta_{j} k_{j} ; v_{1}, \ldots, v_{r}, 0\right),\left(1-\mu-\gamma+\alpha+\alpha^{\prime}-\sum_{j=1}^{s} \lambda_{j} k_{j} ; \sigma_{1}, \ldots, \sigma_{r}, 1\right) \\
& \left(1-\mu-\gamma+\alpha+\beta^{\prime}-\sum_{j=1}^{s} \lambda_{j} k_{j} ; \sigma_{1}, \ldots, \sigma_{r}, 1\right), \\
& \left(1-\mu-\beta-\sum_{j=1}^{s} \lambda_{j} k_{j} ; \sigma_{1}, \ldots, \sigma_{r}, 1\right) .
\end{aligned}
$$

Proof For convenience, let the left-hand side of (2.5) be denoted by $\mathcal{I}$. Applying the Marichev-Saigo-Maeda generalized fractional operators (1.1) to the LHS of (2.5) and making use of (1.15) and (1.6), we obtain

$$
\begin{aligned}
\mathcal{I}= & \left\{\mathrm { I } _ { 0 + } ^ { \alpha , \alpha ^ { \prime } , \beta , \beta ^ { \prime } , \gamma } \left(t^{\mu-1}(b-a t)^{-\gamma} \sum_{k_{1}, \ldots, k_{s}=0}^{m_{1} k_{1}+\cdots+m_{s} k_{s} \leq n} \frac{(-n)_{m_{1} k_{1}+\cdots+m_{s} k_{s}}}{k_{1} ! \cdots k_{s} !} \Lambda\left(n ; k_{1}, \ldots, k_{s}\right)\right.\right. \\
& \times\left(c_{1} t^{\lambda_{1}}(b-a t)^{\delta_{1}}\right)^{k_{1}} \cdots\left(c_{s} t^{\lambda_{s}}(b-a t)^{\delta_{s}}\right)^{k_{s}} \times \frac{1}{(2 \pi i)^{r}} \int_{L_{1}} \cdots \int_{L_{r}} \phi\left(\xi_{1}, \ldots, \xi_{r}\right) \\
& \left.\left.\times \prod_{i=1}^{r} \theta_{i}\left(\xi_{i}\right)\left(z_{i} t^{\sigma_{i}}(b-a t)^{v_{i}}\right)^{\xi_{i}} d \xi_{1} \cdots d \xi_{r}\right)\right\}(x) .
\end{aligned}
$$

By changing the order of summation and integration, we have

$$
\begin{aligned}
\mathcal{I}= & \sum_{k_{1}, \ldots, k_{s}=0}^{m_{1} k_{1}+\cdots+m_{s} k_{s} \leq n} \frac{(-n)_{m_{1} k_{1}+\cdots+m_{s} k_{s}}}{k_{1} ! \cdots k_{s} !} \Lambda\left(n ; k_{1}, \ldots, k_{s}\right) c_{1}^{k_{1}} \cdots c_{s}^{k_{s}} \\
& \times\left[\frac { 1 } { ( 2 \pi i ) ^ { r } } \int _ { L _ { 1 } } \cdots \int _ { L _ { r } } \phi ( \xi _ { 1 } , \ldots , \xi _ { r } ) \prod _ { i = 1 } ^ { r } \{ \theta _ { i } ( \xi _ { i } ) z _ { i } \} ^ { \xi _ { i } } \left\{\mathrm { I } _ { 0 + } ^ { \alpha , \alpha ^ { \prime } , \beta , \beta ^ { \prime } , \gamma } \left(t^{\mu+\sum_{j=1}^{s} \lambda_{j} k_{j}+\sum_{i=1}^{r} \sigma_{i} \xi_{i}-1}\right.\right.\right. \\
& \left.\left.\left.\times(b-a t)^{-\eta-\sum_{j=1}^{s} \lambda_{j} k_{j}-\sum_{i=1}^{r} \sigma_{i} \xi_{i}}\right)\right\}(x) d \xi_{1} \cdots d \xi_{r}\right]
\end{aligned}
$$

By using the binomial expansion for $(b-a t)^{-\eta-\sum_{j=1}^{s} \lambda_{j} k_{j}-\sum_{i=1}^{r} \sigma_{i} \xi_{i}}$ and applying the MellinBarnes counter integral, we get

$$
\begin{aligned}
\mathcal{I}= & \sum_{k_{1}, \ldots, k_{s}=0}^{m_{1} k_{1}+\cdots+m_{s} k_{s} \leq n} \frac{(-n)_{m_{1} k_{1}+\cdots+m_{s} k_{s}}}{k_{1} ! \cdots k_{s} !} \Lambda\left(n ; k_{1}, \ldots, k_{s}\right) c_{1}^{k_{1}} \cdots c_{s}^{k_{s}} b^{-\sum_{j=1}^{s} \lambda_{j} k_{j}} \\
& \times\left[\frac{1}{(2 \pi i)^{r}} \int_{L_{1}} \cdots \int_{L_{r}} \phi\left(\xi_{1}, \ldots, \xi_{r}\right) \times \prod_{i=1}^{r}\left\{\theta_{i}\left(\xi_{i}\right) z_{i} b^{-\sum_{i=1}^{r} \sigma_{i} \xi_{i}}\right\}^{\xi_{i}}\right. \\
& \times \int_{L_{r+1}} \frac{\Gamma\left(\eta+\sum_{j=1}^{s} \lambda_{j} k_{j}+\sum_{i=1}^{r} \sigma_{i} \xi_{i}+\xi_{r+1}\right)}{\Gamma\left(\eta+\sum_{j=1}^{s} \lambda_{j} k_{j}+\sum_{i=1}^{r} \sigma_{i} \xi_{i}\right) \Gamma\left(\xi_{r+1}+1\right)} \\
& \left.\times\left\{\mathrm{I}_{0+}^{\alpha, \alpha^{\prime}, \beta, \beta^{\prime}, \gamma}\left(t^{\mu+\sum_{j=1}^{s} \lambda_{j} k_{j}+\sum_{i=1}^{r} \sigma_{i} \xi_{i}+\xi_{r+1}-1}\right)\right\}(x) d \xi_{1} \cdots d \xi_{r}\right] .
\end{aligned}
$$


Applying (2.1) to (2.9) and re-interpreting the Mellin-Barnes counter integral in terms of the multivariable $H$-function of $r+1$ variables, we obtain the right-hand side of (2.5) after a few simplifications.

Assertion (2.6) of Theorem 2 can be proved in a similar manner by using (2.2) of Lemma 1. So, we omit the details of the proof of Theorem 2.

Here, we derive certain, presumably, new formulas involving Marichev-Saigo-Maeda type fractional integral operators. By setting $n=p=q=0$ in (2.5) and (2.6), respectively, we obtain two fractional integral formulas involving product of the $r, H$-functions stated in Corollaries 1 and 2 below.

Corollary 1 Let $\alpha, \alpha^{\prime}, \beta, \beta^{\prime}, \gamma, \mu, \eta, \delta_{j}, v_{i}, z_{i}, a, b, c_{j} \in \mathbb{C}, \lambda_{j}, \sigma_{i}>0(i \in\{1, \ldots r\} ; j \in\{1, \ldots, s\})$ and $\mathfrak{R}(\gamma)>0$. Then the following relation holds true:

$$
\begin{aligned}
& \left\{\mathrm { I } _ { 0 + } ^ { \alpha , \alpha ^ { \prime } , \beta , \beta ^ { \prime } , \gamma } \left(t^{\mu-1}(b-a t)^{-\eta} S_{n}^{m_{1}, \ldots, m_{s}}\left[c_{1} t^{\lambda_{1}}(b-a t)^{-\delta_{1}}, \ldots, c_{s} t^{\lambda_{s}}(b-a t)^{-\delta_{s}}\right]\right.\right.
\end{aligned}
$$

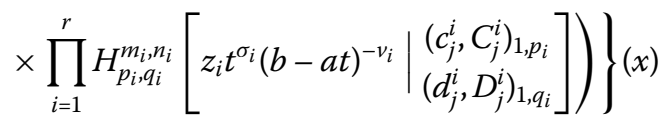

$$
\begin{aligned}
& =b^{-\eta} x^{\mu-\alpha-\alpha^{\prime}+\gamma-1} \sum_{k_{1}, \ldots, k_{s}=0}^{m_{1} k_{1}+\cdots+m_{s} k_{s} \leq n} \frac{(-n)_{m_{1} k_{1}+\cdots+m_{s} k_{s}}}{k_{1} ! \cdots k_{s} !} \Lambda\left(n ; k_{1}, \ldots, k_{s}\right) c_{1}^{k_{1}} \cdots c_{s}^{k_{s}} \\
& \times b^{-\sum_{j=1}^{s} \delta_{j} k_{j}} x^{\sum_{j=1}^{s} \lambda_{j} k_{j}} H_{4,4: p_{i}, q_{i} ; 0,1}^{0,4: m_{i}, n_{i} ; 1,0}\left[\begin{array}{c|c}
z_{i} \frac{x^{\sigma_{i}}}{b^{v_{i}}} & A:\left(c_{j}^{(r)}, \gamma_{j}^{(r)}\right)_{1, p_{i} ;-} \\
\frac{-a}{b} x & B:\left(d_{j}^{(r)}, \delta_{j}^{(r)}\right)_{1, q_{i}} ;(0,1)
\end{array}\right]
\end{aligned}
$$

where $A$ and $B$ are given in Theorem 1 and $H_{p, q}^{m, n}(\cdot)$ is the familiar Fox H-function.

Corollary 2 Let $\alpha, \alpha^{\prime}, \beta, \beta^{\prime}, \gamma, \mu, \eta, \delta_{j}, v_{i}, z_{i}, a, b, c_{j} \in \mathbb{C}, \lambda_{j}, \sigma_{i}>0(i \in\{1, \ldots, r\} ; j \in\{1, \ldots, s\})$ and $\Re(\gamma)>0$. Then the following result holds true:

$$
\begin{aligned}
& \left\{\mathrm { I } _ { 0 - } ^ { \alpha , \alpha ^ { \prime } , \beta , \beta ^ { \prime } , \gamma } \left(t^{\mu-1}(b-a t)^{-\eta} S_{n}^{m_{1}, \ldots, m_{s}}\left[c_{1} t^{\lambda_{1}}(b-a t)^{-\delta_{1}}, \ldots, c_{s} t^{\lambda_{s}}(b-a t)^{-\delta_{s}}\right]\right.\right.
\end{aligned}
$$

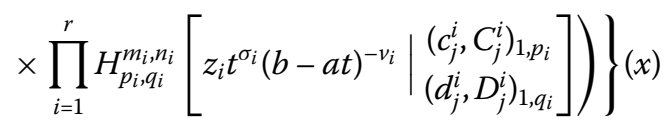

$$
\begin{aligned}
& =b^{-\eta} x^{\mu-\alpha-\alpha^{\prime}+\gamma-1} \sum_{k_{1}, \ldots, k_{s}=0}^{m_{1} k_{1}+\cdots+m_{s} k_{s} \leq n} \frac{(-n)_{m_{1} k_{1}+\cdots+m_{s} k_{s}}}{k_{1} ! \cdots k_{s} !} \Lambda\left(n ; k_{1}, \ldots, k_{s}\right) c_{1}^{k_{1}} \cdots c_{s}^{k_{s}} \\
& \times b^{-\sum_{j=1}^{s} \delta_{j} k_{j}} x^{\sum_{j=1}^{s} \lambda_{j} k_{j}} H_{4,4: p_{i}, q_{i} ; 0,1}^{0,4: m_{i} ; 1,0}\left[\begin{array}{c|c}
z_{i} \frac{x^{\sigma_{i}}}{b^{v_{i}}} & C^{\prime}:\left(c_{j}^{(r)}, \gamma_{j}^{(r)}\right)_{1, p_{i} ;-} \\
\frac{-a}{b} x & D^{\prime}:\left(d_{j}^{(r)}, \delta_{j}^{(r)}\right)_{1, q_{i}} ;(0,1)
\end{array}\right],
\end{aligned}
$$

where

$$
\begin{aligned}
C^{\prime}= & \left(1-\eta-\sum_{j=1}^{s} \delta_{j} k_{j} ; v_{1}, \ldots, v_{r}, 1\right), \\
& \left(1-\mu-\sum_{j=1}^{s} \lambda_{j} k_{j} ; \sigma_{1}, \ldots, \sigma_{r}, 1\right),
\end{aligned}
$$




$$
\begin{aligned}
& \left(1-\mu-\gamma+\alpha+\alpha^{\prime}+\beta^{\prime}-\sum_{j=1}^{s} \lambda_{j} k_{j} ; \sigma_{1}, \ldots, \sigma_{r}, 1\right) \\
& \left(1-\mu+\alpha-\beta-\sum_{j=1}^{s} \lambda_{j} k_{j} ; \sigma_{1}, \ldots, \sigma_{r}, 1\right)
\end{aligned}
$$

and

$$
\begin{aligned}
D^{\prime}= & \left(1-\eta-\sum_{j=1}^{s} \delta_{j} k_{j} ; v_{1}, \ldots, v_{r}, 0\right),\left(1-\mu-\gamma+\alpha+\alpha^{\prime}-\sum_{j=1}^{s} \lambda_{j} k_{j} ; \sigma_{1}, \ldots, \sigma_{r}, 1\right), \\
& \left(1-\mu-\gamma+\alpha+\beta^{\prime}-\sum_{j=1}^{s} \lambda_{j} k_{j} ; \sigma_{1}, \ldots, \sigma_{r}, 1\right) \\
& \left(1-\mu-\beta-\sum_{j=1}^{s} \lambda_{j} k_{j} ; \sigma_{1}, \ldots, \sigma_{r}, 1\right)
\end{aligned}
$$

where $H_{p, q}^{m, n}(\cdot)$ is the familiar Fox H-function.

Interestingly, in view of relation (1.4), we arrive at the following corollaries concerning Saigo fractional integral operator. It is similar to the results due to Agarwal [47], p.587 and p.590, Eqs. (18) and (25).

Corollary 3 Let $\alpha, \beta, \gamma, \mu, \eta, \delta_{j}, v_{i}, z_{i}, a, b, c_{j} \in \mathbb{C}, \lambda_{j}, \sigma_{i}>0(i \in\{1, \ldots, r\} ; j \in\{1, \ldots, s\})$, and $\Re(\alpha)>0$. Let the following conditions be satisfied, too:

(i) $\left|\arg z_{i}\right|<\frac{\pi}{2} \Omega_{i}, \Omega_{i}>0(i=1, \ldots, r)$, where

$$
\begin{aligned}
\Omega_{i}= & -\sum_{j=n+1}^{p} \alpha_{j}^{(i)}-\sum_{j=1}^{q} \beta_{j}^{(i)}+\sum_{j=1}^{n_{i}} \gamma_{j}^{(i)}-\sum_{j=n_{i}+1}^{p_{i}} \gamma_{j}^{(i)} \\
& +\sum_{j=1}^{m_{i}} \delta_{j}^{(i)}-\sum_{j=m_{i}+1}^{q_{i}} \delta_{j}^{(i)}>0 \quad(i \in\{1, \ldots, r\}) .
\end{aligned}
$$

(ii)

$$
\begin{aligned}
& \Re(\mu)+\sum_{i=1}^{r} \sigma_{i} \min _{1 \leq j \leq m_{i}}\left[\frac{\Re\left(d_{j}^{(i)}\right)}{\delta_{j}^{(i)}}\right]>\max [0, \Re(\beta-\gamma)], \\
& \Re(\eta)+\sum_{i=1}^{r} v_{i} \min _{1 \leq j \leq m_{i}}\left[\frac{\Re\left(d_{j}^{(i)}\right)}{\delta_{j}^{(i)}}\right]>\max [0, \Re(\beta-\gamma)] \text { and }\left|\frac{a}{b} x\right|<1 .
\end{aligned}
$$

Then the following result holds:

$$
\begin{aligned}
& \left\{\mathrm { I } _ { 0 + } ^ { \alpha , \beta , \gamma } \left(t^{\mu-1}(b-a t)^{-\eta} S_{n}^{m_{1}, \ldots, m_{s}}\left[c_{1} t^{\lambda_{1}}(b-a t)^{-\delta_{1}}, \ldots, c_{s} t^{\lambda_{s}}(b-a t)^{-\delta_{s}}\right]\right.\right. \\
& \left.\left.\quad \times H\left[z_{1} t^{\sigma_{1}}(b-a t)^{-\nu_{1}}, \ldots, z_{r} t^{\sigma_{r}}(b-a t)^{-v_{r}}\right]\right)\right\}(x) \\
& =b^{-\eta} x^{\mu-\beta-1} \sum_{k_{1}, \ldots, k_{s}=0}^{m_{1} k_{1}+\cdots+m_{s} k_{s} \leq n} \frac{(-n)_{m_{1} k_{1}+\cdots+m_{s} k_{s}}}{k_{1} ! \cdots k_{s} !} \Lambda\left(n ; k_{1}, \ldots, k_{s}\right) c_{1}^{k_{1}} \cdots c_{s}^{k_{s}}
\end{aligned}
$$




$$
\begin{aligned}
& \times b^{-\sum_{j=1}^{s} \delta_{j} k_{j}} x^{\sum_{j=1}^{s} \lambda_{j} k_{j}} H_{p+3, q+3:\left\{p_{r}, q_{r}\right\} ; 0,1}^{0, n+3:\left\{m_{r}, n_{r}\right\} ; 1,0} \\
& {\left[\begin{array}{c|c}
z_{1} \frac{x^{\sigma_{1}}}{b^{v_{1}}} & \left(a_{j} ; \alpha_{j}^{(1)}, \ldots, \alpha_{j}^{(r)}\right)_{1, p}, A^{\prime}:\left\{\left(c_{j}^{(r)}, \gamma_{j}^{(r)}\right)_{1, p_{r}}\right\} ;- \\
\vdots & \left(b_{j} ; \beta_{j}^{(1)}, \ldots, \beta_{j}^{(r)}\right)_{1, q}, B^{\prime}:\left\{\left(d_{j}^{(r)}, \delta_{j}^{(r)}\right)_{1, q_{r}}\right\} ;(0,1) \\
z_{r} \frac{x^{\sigma_{r}}}{b^{v_{r}}} & \\
-\frac{a}{b} x &
\end{array}\right],}
\end{aligned}
$$

where

$$
\begin{aligned}
A^{\prime}= & \left(1-\eta-\sum_{j=1}^{s} \delta_{j} k_{j} ; v_{1}, \ldots, v_{r}, 1\right),\left(1-\mu-\sum_{j=1}^{s} \lambda_{j} k_{j} ; \sigma_{1}, \ldots, \sigma_{r}, 1\right), \\
& \left(1-\mu-\gamma+\beta-\sum_{j=1}^{s} \lambda_{j} k_{j} ; \sigma_{1}, \ldots, \sigma_{r}, 1\right)
\end{aligned}
$$

and

$$
\begin{aligned}
B^{\prime}= & \left(1-\eta-\sum_{j=1}^{s} \delta_{j} k_{j} ; v_{1}, \ldots, v_{r}, 0\right),\left(1-\mu+\beta-\sum_{j=1}^{s} \lambda_{j} k_{j} ; \sigma_{1}, \ldots, \sigma_{r}, 1\right) \\
& \left(1-\mu-\gamma-\alpha-\sum_{j=1}^{s} \lambda_{j} k_{j} ; \sigma_{1}, \ldots, \sigma_{r}, 1\right)
\end{aligned}
$$

and the conditions of existence of the above corollary follow easily from Theorem 1.

Corollary 4 Let $\alpha, \beta, \gamma, \mu, \eta, \delta_{j}, v_{i}, z_{i}, a, b, c_{j} \in \mathbb{C}, \lambda_{j}, \sigma_{i}>0(i \in\{1, \ldots, r\} ; j \in\{1, \ldots, s\})$, and $\Re(\alpha)>0$. Let the following conditions be satisfied, too:

(i) $\left|\arg z_{i}\right|<\frac{\pi}{2} \Omega_{i}, \Omega_{i}>0(i=1, \ldots, r)$, where

$$
\Omega_{i}=-\sum_{j=n+1}^{p} \alpha_{j}^{(i)}-\sum_{j=1}^{q} \beta_{j}^{(i)}+\sum_{j=1}^{n_{i}} \gamma_{j}^{(i)}-\sum_{j=n_{i}+1}^{p_{i}} \gamma_{j}^{(i)}+\sum_{j=1}^{m_{i}} \delta_{j}^{(i)}-\sum_{j=m_{i}+1}^{q_{i}} \delta_{j}^{(i)}>0 .
$$

(ii)

$$
\begin{aligned}
& \Re(\mu)-\sum_{i=1}^{r} \sigma_{i} \min _{1 \leq j \leq m_{i}}\left[\frac{\Re\left(d_{j}^{(i)}\right)}{\delta_{j}^{(i)}}\right]<1+\max [\Re(\beta), \Re(\gamma)] \quad(i=1, \ldots, r), \\
& \Re(\eta)-\sum_{i=1}^{r} v_{i} \min _{1 \leq j \leq m_{i}}\left[\frac{\Re\left(d_{j}^{(i)}\right)}{\delta_{j}^{(i)}}\right]<1+\max [\Re(\beta), \Re(\gamma)] \quad(i=1, \ldots, r) .
\end{aligned}
$$

Then the following result holds:

$$
\begin{aligned}
& \left\{\mathrm { I } _ { 0 - } ^ { \alpha , \beta , \gamma } \left(t^{\mu-1}(b-a t)^{-\eta} S_{n}^{m_{1}, \ldots, m_{s}}\left[c_{1} t^{\lambda_{1}}(b-a t)^{-\delta_{1}}, \ldots, c_{s} t^{\lambda_{s}}(b-a t)^{-\delta_{s}}\right]\right.\right. \\
& \left.\left.\quad \times H\left[z_{1} t^{\sigma_{1}}(b-a t)^{-v_{1}}, \ldots, z_{r} t^{\sigma_{r}}(b-a t)^{-v_{r}}\right]\right)\right\}(x) \\
& =b^{-\eta} x^{\mu-\beta-1} \sum_{k_{1}, \ldots, k_{s}=0}^{m_{1} k_{1}+\cdots+m_{s} k_{s} \leq n} \frac{(-n)_{m_{1} k_{1}+\cdots+m_{s} k_{s}}}{k_{1} ! \cdots k_{s} !} \Lambda\left(n ; k_{1}, \ldots, k_{s}\right) c_{1}^{k_{1}} \cdots c_{s}^{k_{s}}
\end{aligned}
$$




$$
\begin{aligned}
& \times b^{-\sum_{j=1}^{s} \delta_{j} k_{j}} x^{\sum_{j=1}^{s} \lambda_{j} k_{j}} H_{p+3, q+3:\left\{p_{r}, q_{r}\right\} ; 0,1}^{0, n+3:\left\{m_{r}, n_{r}\right\} ; 1,0} \\
& {\left[\begin{array}{c|c}
z_{1} \frac{\chi^{\sigma_{1}}}{b^{v_{1}}} & \left(a_{j} ; \alpha_{j}^{(1)}, \ldots, \alpha_{j}^{(r)}\right)_{1, p}, C^{\prime \prime}:\left\{\left(c_{j}^{(r)}, \gamma_{j}^{(r)}\right)_{1, p_{r}}\right\} ;- \\
\vdots & \left(b_{j} ; \beta_{j}^{(1)}, \ldots, \beta_{j}^{(r)}\right)_{1, q}, D^{\prime \prime}:\left\{\left(d_{j}^{(r)}, \delta_{j}^{(r)}\right)_{1, q_{r}}\right\} ;(0,1) \\
z_{r} \frac{x^{\sigma_{r}}}{b^{v_{r}}} & \left(b_{j}\right) \\
-\frac{a}{b} x &
\end{array}\right]}
\end{aligned}
$$

where

$$
\begin{aligned}
C^{\prime \prime}= & \left(1-\eta-\sum_{j=1}^{s} \delta_{j} k_{j} ; v_{1}, \ldots, v_{r}, 1\right),\left(1-\mu-\sum_{j=1}^{s} \lambda_{j} k_{j} ; \sigma_{1}, \ldots, \sigma_{r}, 1\right), \\
& \left(1+\alpha+\beta-\mu-\gamma-\sum_{j=1}^{s} \lambda_{j} k_{j} ; \sigma_{1}, \ldots, \sigma_{r}, 1\right)
\end{aligned}
$$

and

$$
\begin{aligned}
D^{\prime \prime}= & \left(1-\eta-\sum_{j=1}^{s} \delta_{j} k_{j} ; v_{1}, \ldots, v_{r}, 0\right),\left(1-\gamma-\mu-\sum_{j=1}^{s} \lambda_{j} k_{j} ; \sigma_{1}, \ldots, \sigma_{r}, 1\right) \\
& \left(1+\beta-\mu-\sum_{j=1}^{s} \lambda_{j} k_{j} ; \sigma_{1}, \ldots, \sigma_{r}, 1\right),
\end{aligned}
$$

and the conditions of existence of the above corollary follow easily from Theorem 2.

Remark 5 By putting $\beta=-\alpha$ and $\beta=0$ in Corollaries 3 and 4, respectively, we can obtain very interesting results involving the Riemann-Liouville and Erdélyi-Kober fractional integral operators.

\section{Special cases and concluding remarks}

Here we consider another variation of the results derived in the preceding sections. The multivariable $H$-function occurring in these results can be suitably specialized to yield a wide variety of special functions (or product of such functions) of one or more variables (see [31], pp.18-19, 88-93, 253-254 and [48]). Again, by suitably specializing the coefficient of the first class of multivariable polynomials, it can be reduced to other multivariable hypergeometric polynomials and classical orthogonal polynomials of one or more variables.

(i) If we reduce the multivariable $H$-functions into the product of two Fox $H$-functions in Theorem 1 and then reduce one $H$-function to the exponential function by taking $\sigma_{1}=1$, $v_{1} \rightarrow 0$, we obtain the following result after a little simplification:

$$
\begin{aligned}
& \left\{\mathrm { I } _ { 0 + } ^ { \alpha , \alpha ^ { \prime } , \beta , \beta ^ { \prime } , \gamma } \left(t^{\mu-1}(b-a t)^{-\eta} S_{n}^{m_{1}, \ldots, m_{s}}\left[c_{1} t^{\lambda_{1}}(b-a t)^{-\delta_{1}}, \ldots, c_{s} t^{\lambda_{s}}(b-a t)^{-\delta_{s}}\right]\right.\right. \\
& \left.\left.\quad \times e^{-z_{1} t} H_{p_{2}, q_{2}}^{m_{2}, n_{2}}\left[z_{2} t^{\sigma_{2}}(b-a t)^{-\nu_{2}} \mid \begin{array}{c}
\left(c_{j}, C_{j}\right)_{1, p_{2}} \\
\left(d_{j}, D_{j}\right)_{1, q_{2}}
\end{array}\right]\right)\right\}(x) \\
& =b^{-\eta} x^{\mu-\alpha-\alpha^{\prime}+\gamma-1} \sum_{k_{1}, \ldots, k_{s}=0}^{m_{1} k_{1}+\cdots+m_{s} k_{s} \leq n} \frac{(-n)_{m_{1} k_{1}+\cdots+m_{s} k_{s}}}{k_{1} ! \cdots k_{s} !} \Lambda\left(n ; k_{1}, \ldots, k_{s}\right) c_{1}^{k_{1}} \cdots c_{s}^{k_{s}}
\end{aligned}
$$




$$
\begin{aligned}
& \times b^{-\sum_{j=1}^{s} \delta_{j} k_{j}} x^{\sum_{j=1}^{s} \lambda_{j} k_{j}} H_{4,4: 0,1 ; ; p_{2}, q_{2} ; 0,1}^{0,4: 1, m_{2}, n_{2} ; 1,0} \\
& {\left[\begin{array}{c|c}
z_{1} x & E:\left(c_{j}, \gamma_{j}\right)_{1, p_{2}} ;- \\
z_{2} \frac{x^{\sigma_{2}}}{b^{v_{2}}} & F:(0,1) ;\left\{\left(d_{j}, \delta_{j}\right)_{1, q_{2}}\right\} ;(0,1) \\
-\frac{a}{b} x &
\end{array}\right],}
\end{aligned}
$$

where

$$
\begin{aligned}
E= & \left(1-\eta-\sum_{j=1}^{s} \delta_{j} k_{j} ; 1, \nu_{2}, 1\right),\left(1-\mu-\sum_{j=1}^{s} \lambda_{j} k_{j} ; 1, \sigma_{2}, 1\right) \\
& \left(1-\mu-\gamma+\alpha+\alpha^{\prime}+\beta-\sum_{j=1}^{s} \lambda_{j} k_{j} ; 1, \sigma_{2}, 1\right) \\
& \left(1-\mu+\alpha^{\prime}-\beta^{\prime}-\sum_{j=1}^{s} \lambda_{j} k_{j} ; 1, \sigma_{2}, 1\right) \\
F= & \left(1-\eta-\sum_{j=1}^{s} \delta_{j} k_{j} ; 1, \nu_{2}, 0\right),\left(1-\mu-\gamma+\alpha+\alpha-\sum_{j=1}^{s} \lambda_{j} k_{j} ; 1, \sigma_{2}, 1\right) \\
& \left(1-\mu-\gamma+\alpha^{\prime}+\beta-\sum_{j=1}^{s} \lambda_{j} k_{j} ; 1, \sigma_{2}, 1\right),\left(1-\mu-\beta^{\prime}-\sum_{j=1}^{s} \lambda_{j} k_{j} ; 1, \sigma_{2}, 1\right) .
\end{aligned}
$$

The conditions of validity of the above result easily follow from Theorem 1 .

Remark 6 If we reduce the Marichev-Saigo-Maeda type fractional integral operator into the Riemann-Liouville fractional integral operator and put $S_{n}^{m_{1}, \ldots, m_{s}}=1, \eta, v_{2}=0$, and make a suitable adjustment in the parameters in the above equation, then we obtain the known result by Kilbas and Saigo [49], p.52, Eq. (2.7.9); in addition, if we put $S_{n_{j}}^{m_{j}}=1, \eta, v_{2}=0$, $z_{2}=1 / 4, \sigma_{2}=2$ and reduce the Fox $H$-function to the Bessel function of the first kind, then we obtain the known result given by Kilbas and Sebastian [50], p.873, Eqs. (25)-(29).

(ii) Next if we take $z_{2}, \sigma_{2}=1$ and $v_{2}=0$ in the result (3.1) and reduce the $H$-function of one variable to a generalized Mittag-Leffler function [51] (see also [52]), we can easily obtain the following result after a few simplifications:

$$
\begin{aligned}
& \left\{\mathrm { I } _ { 0 + } ^ { \alpha , \alpha ^ { \prime } , \beta , \beta ^ { \prime } , \gamma } \left(t^{\mu-1}(b-a t)^{-\eta} S_{n}^{m_{1}, \ldots, m_{s}}\left[c_{1} t^{\lambda_{1}}(b-a t)^{-\delta_{1}}, \ldots, c_{s} t^{\lambda_{s}}(b-a t)^{-\delta_{s}}\right]\right.\right. \\
& \left.\left.\times e^{-z_{1} t} E_{\omega, \xi}^{v}[t]\right)\right\}(x) \\
& =\frac{b^{-\eta} x^{\mu-\alpha-\alpha^{\prime}+\gamma-1}}{\Gamma(v)} \sum_{k_{1}, \ldots, k_{s}=0}^{m_{1} k_{1}+\cdots+m_{s} k_{s} \leq n} \frac{(-n)_{m_{1} k_{1}+\cdots+m_{s} k_{s}}}{k_{1} ! \cdots k_{s} !} \Lambda\left(n ; k_{1}, \ldots, k_{s}\right) c_{1}^{k_{1}} \cdots c_{s}^{k_{s}} \\
& \times b^{-\sum_{j=1}^{s} \delta_{j} k_{j}}(-x)^{\sum_{j=1}^{s} \lambda_{j} k_{j}} H_{4,3: 0,1 ; 1,3 ; 0,1}^{0,4: 1,0 ; 1,1 ;, 0} \\
& {\left[\begin{array}{c|c}
z_{1} x & E:-;(1-v, 1) ;- \\
x & F:(0,1) ;(0,1),(1-\eta ; 0) ;(1-\xi, \omega) ;(0,1) \\
-\frac{a}{b} x &
\end{array}\right]}
\end{aligned}
$$


where

$$
\begin{aligned}
E= & \left(1-\eta-\sum_{j=1}^{s} \delta_{j} k_{j} ; 1,0,1\right),\left(1-\mu-\sum_{j=1}^{s} \lambda_{j} k_{j} ; 1,1,1\right), \\
& \left(1-\mu-\gamma+\alpha+\alpha^{\prime}+\beta-\sum_{j=1}^{s} \lambda_{j} k_{j} ; 1,1,1\right),\left(1-\mu+\alpha^{\prime}-\beta^{\prime}-\sum_{j=1}^{s} \lambda_{j} k_{j} ; 1,1,1\right), \\
F= & \left(1-\eta-\sum_{j=1}^{s} \delta_{j} k_{j} ; 1,0,0\right),\left(1-\mu-\gamma+\alpha+\alpha^{\prime}-\sum_{j=1}^{s} \lambda_{j} k_{j} ; 1,1,1\right), \\
& \left(1-\mu-\gamma+\alpha^{\prime}+\beta-\sum_{j=1}^{s} \lambda_{j} k_{j} ; 1,1,1\right),\left(1-\mu-\beta^{\prime}-\sum_{j=1}^{s} \lambda_{j} k_{j} ; 1,1,1\right) .
\end{aligned}
$$

The conditions of validity of (3.2) can be easily followed directly by those given with (2.5).

Remark 7 If we reduce the Marichev-Saigo-Maeda type fractional integral operator to the Riemann-Liouville fractional integral operator and set $S_{n}^{m_{1}, \ldots, m_{s}}=1, \eta, v_{2}=0$, and make a suitable adjustment in the parameters in equation (3.2), then we arrive at the known result given by Saxena et al. [53], p.168, Eq. (2.1).

(iii) Again, if we reduce the $H$-function of one variable to the generalized Wright hypergeometric function [31], p.19, Eq. (2.6.11), in the result (3.1), we arrive at the following new and interesting result:

$$
\begin{aligned}
& \left\{\mathrm { I } _ { 0 + } ^ { \alpha , \alpha ^ { \prime } , \beta , \beta ^ { \prime } , \gamma } \left(t^{\mu-1}(b-a t)^{-\eta} S_{n}^{m_{1}, \ldots, m_{s}}\left[c_{1} t^{\lambda_{1}}(b-a t)^{-\delta_{1}}, \ldots, c_{s} t^{\lambda_{s}}(b-a t)^{-\delta_{s}}\right]\right.\right. \\
& \left.\left.\times e^{-z_{1} t}{ }_{p_{2}} \psi_{q_{2}}\left[\begin{array}{l|c}
-z_{2} t^{\sigma_{2}}(b-a t)^{-v_{2}} & \left(1-c_{j}, C_{j}\right)_{1, p_{2}} \\
(0,1),\left(1-d_{j}, D_{j}\right)_{1, q_{2}}
\end{array}\right]\right)\right\}(x) \\
& =b^{-\eta} x^{\mu-\alpha-\alpha^{\prime}+\gamma-1} \sum_{k_{1}, \ldots, k_{s}=0}^{m_{1} k_{1}+\cdots+m_{s} k_{s} \leq n} \frac{(-n)_{m_{1} k_{1}+\cdots+m_{s} k_{s}}}{k_{1} ! \cdots k_{s} !} \Lambda\left(n ; k_{1}, \ldots, k_{s}\right) c_{1}^{k_{1}} \cdots c_{s}^{k_{s}} \\
& \times b^{-\sum_{j=1}^{S} \delta_{j} k_{j}} x^{\sum_{j=1}^{S} \lambda_{j} k_{j}} H_{4,4: 0,1 ; p_{2}, q_{2} ; 0,1}^{0,4: 1,0 ; 1, p_{2} ; 1,0} \\
& {\left[\begin{array}{c|c}
z_{1} x & E:\left(c_{j}, C_{j}\right)_{1, p_{2}} ;- \\
-z_{2} \frac{x^{\sigma_{2}}}{b^{\nu 2}} & F:(0,1) ;\left\{\left(d_{j}, D_{j}\right)_{1, q_{2}}\right\} ;(0,1) \\
-\frac{a}{b} x &
\end{array}\right]}
\end{aligned}
$$

where

$$
\begin{aligned}
E= & \left(1-\eta-\sum_{j=1}^{s} \delta_{j} k_{j} ; 1, v_{2}, 1\right),\left(1-\mu-\sum_{j=1}^{s} \lambda_{j} k_{j} ; 1, \sigma_{2}, 1\right), \\
& \left(1-\mu-\gamma+\alpha+\alpha^{\prime}+\beta-\sum_{j=1}^{s} \lambda_{j} k_{j} ; 1, \sigma_{2}, 1\right),\left(1-\mu+\alpha^{\prime}-\beta^{\prime}-\sum_{j=1}^{s} \lambda_{j} k_{j} ; 1, \sigma_{2}, 1\right),
\end{aligned}
$$




$$
\begin{aligned}
F= & \left(1-\eta-\sum_{j=1}^{s} \delta_{j} k_{j} ; 1, v_{2}, 0\right),\left(1-\mu-\gamma+\alpha+\alpha^{\prime}-\sum_{j=1}^{s} \lambda_{j} k_{j} ; 1, \sigma_{2}, 1\right), \\
& \left(1-\mu-\gamma+\alpha^{\prime}+\beta-\sum_{j=1}^{s} \lambda_{j} k_{j} ; 1, \sigma_{2}, 1\right),\left(1-\mu-\beta^{\prime}-\sum_{j=1}^{s} \lambda_{j} k_{j} ; 1, \sigma_{2}, 1\right) .
\end{aligned}
$$

(iv) Further Theorem 1, if we reduce the multivariable $H$-function to the product of $r$-different Whittaker functions [31], p.18, Eq. (2.6.7), and take $v_{i} \rightarrow 0, \sigma_{i}=1$, then we arrive at the following result:

$$
\begin{aligned}
& \left\{\mathrm { I } _ { 0 + } ^ { \alpha , \alpha ^ { \prime } , \beta , \beta ^ { \prime } , \gamma } \left(t^{\mu-1}(b-a t)^{-\eta} S_{n}^{m_{1}, \ldots, m_{s}}\left[c_{1} t^{\lambda_{1}}(b-a t)^{-\delta_{1}}, \ldots, c_{s} t^{\lambda_{s}}(b-a t)^{-\delta_{s}}\right]\right.\right. \\
& \left.\left.\times \prod_{i=1}^{r} e^{\frac{-z_{i} t}{2}} W_{\lambda_{i}, \mu_{i}}\left(z_{i} t\right)\right)\right\}(x) \\
& =b^{-\eta} x^{\mu-\alpha-\alpha^{\prime}+\gamma-1} \sum_{k_{1}, \ldots, k_{s}=0}^{m_{1} k_{1}+\cdots+m_{s} k_{s} \leq n} \frac{(-n)_{m_{1} k_{1}+\cdots+m_{s} k_{s}}}{k_{1} ! \cdots k_{s} !} \Lambda\left(n ; k_{1}, \ldots, k_{s}\right) c_{1}^{k_{1}} \cdots c_{s}^{k_{s}} \\
& \times b^{-\sum_{j=1}^{s} \delta_{j} k_{j}} x^{\sum_{j=1}^{s} \lambda_{j} k_{j}} H_{4,4: 1,2 ; \ldots ; 1,2 ; 0,1}^{0,4: 2,0 ; \ldots 2,0 ; 1,0} \\
& {\left[\begin{array}{c|c}
z_{1} x & \\
\vdots & G:-;\left(1-\lambda_{1}, 1\right) ; \ldots ;\left(1-\lambda_{r}, 1\right) ;- \\
z_{r} x & H:\left(1 / 2 \pm \mu_{1}, 1\right) ; \ldots ;\left(1 / 2 \pm \mu_{r}, 1\right)(0,1) \\
-\frac{a}{b} x &
\end{array}\right],}
\end{aligned}
$$

where

$$
\begin{aligned}
G= & (1-\eta-\sum_{j=1}^{s} \delta_{j} k_{j} ; \underbrace{1, \ldots, 1}_{r \text { times }}, 1),(1-\mu-\sum_{j=1}^{s} \lambda_{j} k_{j} ; \underbrace{1, \ldots, 1}_{r \text { times }}, 1), \\
& (1-\mu-\gamma+\alpha+\alpha^{\prime}+\beta-\sum_{j=1}^{s} \lambda_{j} k_{j} ; \underbrace{1, \ldots, 1}_{r \text { times }}, 1), \\
& (1-\mu+\alpha^{\prime}-\beta^{\prime}-\sum_{j=1}^{s} \lambda_{j} k_{j} ; \underbrace{1, \ldots, 1}_{r \text { times }}, 1), \\
H= & (1-\eta-\sum_{j=1}^{s} \delta_{j} k_{j} ; \underbrace{1, \ldots, 1}_{r \text { times }}, 0),(1-\mu-\gamma+\alpha+\alpha^{\prime}-\sum_{j=1}^{s} \lambda_{j} k_{j} ; \underbrace{1, \ldots, 1}_{r \text { times }}, 1), \\
& (1-\mu-\gamma+\alpha^{\prime}+\beta-\sum_{j=1}^{s} \lambda_{j} k_{j} ; \underbrace{1, \ldots, 1}_{r \text { times }}, 1),(1-\mu-\beta^{\prime}-\sum_{j=1}^{s} \lambda_{j} k_{j} ; \underbrace{1, \ldots, 1}_{r \text { times }}, 1) .
\end{aligned}
$$

The conditions of validity of the above result easily follow from Theorem 1 .

Remark 8 If we reduce the Marichev-Saigo-Maeda type fractional integral operator to the Riemann-Liouville fractional integral operator and set $S_{n}^{m_{1}, \ldots, m_{s}}=1, \eta, v_{2}=0$, and make a suitable adjustment in the parameters in equation (3.4), we arrive at the known result given by Kilbas [54], p.117, Eq. (11). 
(v) Finally, if we reduce the multivariable $H$-function into the product of two Fox $H$-functions in Theorem 1 and then reduce one $H$-function to the exponential function by setting $\sigma_{1}, \lambda_{j}, v_{1}, \delta_{j}=0, c_{j}, s=1$ and $S_{n}^{m}$ to the Hermite polynomial $[6,55]$, and we set $S_{n}^{2}[x]=x^{n / 2} H_{n}\left[\frac{1}{2 \sqrt{x}}\right]$, in this case $m=2, A_{n, k}=(-1)^{k}$, we obtain the following interesting result:

$$
\begin{aligned}
& \left\{\mathrm { I } _ { 0 + } ^ { \alpha , \alpha ^ { \prime } , \beta , \beta ^ { \prime } , \gamma } \left(t^{\mu-1}(b-a t)^{-\eta} t^{n / 2} H_{n}\left[\frac{1}{2 \sqrt{t}}\right]\right.\right. \\
& \left.\left.\times e^{-z_{1} t} H_{p_{2}, q_{2}}^{m_{2}, n_{2}}\left[\begin{array}{l|l}
z_{1} t^{\sigma_{1}}(b-a t)^{-v_{1}} & \begin{array}{l}
\left(c_{j}, C_{j}\right)_{1, p_{2}} \\
\left(d_{j}, D_{j}\right)_{1, q_{2}}
\end{array}
\end{array}\right]\right)\right\}(x) \\
& =b^{-\eta} x^{\mu-\alpha-\alpha^{\prime}+\gamma-1} \sum_{k_{1}, \ldots, k_{s}=0}^{m_{1} k_{1}+\cdots+m_{s} k_{s} \leq n} \frac{(-n)_{m_{1} k_{1}+\cdots+m_{s} k_{s}}}{k_{1} ! \cdots k_{s} !} \Lambda\left(n ; k_{1}, \ldots, k_{s}\right) c_{1}^{k_{1}} \cdots c_{s}^{k_{s}} \\
& \times b^{-\sum_{j=1}^{s} \delta_{j} k_{j}} x^{\sum_{j=1}^{s} \lambda_{j} k_{j}} H_{4,4: 0,0, ;\left\{p_{2}, q_{2}\right\} ; ; 0,1}^{\left.0,4: 0 ; m_{2}, n_{2}\right\} ; 1,0} \\
& {\left[\begin{array}{c|c}
z_{1} x & G:\left(c_{j}^{(r)}, \gamma_{j}^{(r)}\right)_{1, p_{r}} ;- \\
z_{2} \frac{x^{\sigma_{2}}}{b^{\nu_{2}}} & H:(0,1) ;\left\{\left(d_{j}, \delta_{j}\right)_{1, q_{2}}\right\} ;(0,1) \\
-\frac{a}{b} x &
\end{array}\right]}
\end{aligned}
$$

where

$$
\begin{aligned}
G= & \left(1-\eta-\sum_{j=1}^{s} \delta_{j} k_{j} ; 1, \nu_{2}, 1\right),\left(1-\mu-\sum_{j=1}^{s} \lambda_{j} k_{j} ; 1, \sigma_{2}, 1\right), \\
& \left(1-\mu-\gamma+\alpha+\alpha^{\prime}+\beta-\sum_{j=1}^{s} \lambda_{j} k_{j} ; 1, \sigma_{2}, 1\right),\left(1-\mu+\alpha^{\prime}-\beta^{\prime}-\sum_{j=1}^{s} \lambda_{j} k_{j} ; 1, \sigma_{2}, 1\right), \\
H= & \left(1-\eta-\sum_{j=1}^{s} \delta_{j} k_{j} ; 1, \nu_{2}, 0\right),\left(1-\mu-\gamma+\alpha+\alpha-\sum_{j=1}^{s} \lambda_{j} k_{j} ; 1, \sigma_{2}, 1\right), \\
& \left(1-\mu-\gamma+\alpha^{\prime}+\beta-\sum_{j=1}^{s} \lambda_{j} k_{j} ; 1, \sigma_{2}, 1\right),\left(1-\mu-\beta^{\prime}-\sum_{j=1}^{s} \lambda_{j} k_{j} ; 1, \sigma_{2}, 1\right) .
\end{aligned}
$$

The conditions of validity of the above result can be easily derived from Theorem 1 .

A large number of other special cases of our main result can also be obtained, but we do not mention them here on account of the lack of space.

Now, we conclude our present investigation by remarking that the fractional integration (of Marichev-Saigo-Maeda type) of the products of multivariable $H$-functions and the first class of multivariable polynomials established in this paper will be useful for investigators in various disciplines of applied sciences and engineering physics. We are also trying to find certain possible applications of those results presented here to some other research areas, for example, obtaining a closed form solution of a fractional generalization of a free electron equation. 


\section{Authors' contributions}

All authors have participated in the obtained results. The collaboration of each one cannot be separated in different parts of the paper. All of them have made substantial contributions to the theoretical results. All authors have been involved in drafting the manuscript and revising it critically for important intellectual content. All authors read and approved the final manuscript.

\section{Author details}

${ }^{1}$ Department of Mathematics, Anand International College of Engineering, Jaipur, 303012, India. ${ }^{2}$ Department of Economics, Belarusian State University, Minsk, 220030, Belarus. ${ }^{3}$ Institute of Mathematics, National University of Uzbekistan, Tashkent, 100125, Uzbekistan. ${ }^{4}$ Department of Mathematics, Fateh College for Women, Bathinda, 151103, India.

\section{Acknowledgements}

We are grateful to Dr. S Jain for her useful suggestions during discussions and to anonymous reviewers for their valuable remarks.

\section{Endnote}

a Here and in what follows, we denote by $\omega$ an imaginary unit.

Received: 22 July 2015 Accepted: 30 October 2015 Published online: 05 November 2015

\section{References}

1. Baleanu, D, Diethelm, K, Scalas, E, Trujillo, JJ: Fractional Calculus: Models and Numerical Methods. World Scientific, Singapore (2012)

2. Caponetto, R, Dongola, G, Fortuna, L, Petráš, I: Fractional Order Systems: Modeling and Control Applications. World Scientific, Singapore (2010)

3. Caputo, M, Mainardi, F: Linear models of dissipation in anelastic solids. Riv. Nuovo Cimento, Ser. II 1, 161-198 (1971)

4. Hilfer, R (ed.): Applications of Fractional Calculus in Physics. Word Scientific, River Edge (2000)

5. Mainardi, F: Fractional Calculus and Waves in Linear Viscoelasticity. Imperial College Press, London (2010)

6. Mathai, AM, Saxena, RK, Haubold, HJ: The H-Function: Theory and Applications. Springer, Dordrecht (2010)

7. Prakasa Rao, BLS: Statistical Inference for Fractional Diffusion Processes. Wiley, Chichester (2010)

8. Rabotnov, YN: Elements of Hereditary Solid Mechanics. Mir, Moscow (1980)

9. Tarasov, VE: Fractional Dynamics: Application of Fractional Calculus to Dynamics of Particles, Fields and Media. Springer, Berlin (2010)

10. Uchaikin, W: Fractional Derivatives for Physicists and Engineers. Volume I: Background and Theory. Springer, Berlin (2013)

11. Uchaikin, V: Fractional Derivatives for Physicists and Engineers. Volume II: Applications. Springer, Berlin (2013)

12. Zaslavsky, GM: Hamiltonian Chaos and Fractional Dynamics. Oxford University Press, Oxford (2005)

13. Marichev, OI: Volterra equation of Mellin convolution type with a Horn function in the kernel. Izv. Akad. Nauk BSSR, Ser. Fiz.-Mat. Nauk 1, 128-129 (1974)

14. Saigo, M, Maeda, N: More generalization of fractional calculus. In: Rusev, P, Dimovski, l, Kiryakova, V (eds.) Transform Methods and Special Functions, Varna, 1996 (Proc. 2nd Intern. Workshop), pp. 386-400. IMI-BAS, Sofia (1998)

15. Saxena, RK, Saigo, M: Generalized fractional calculus of the $H$-function associated with the Appell function $F_{3}$. J. Fract. Calc. 19, 89-104 (2001)

16. Agarwal, P: Generalized fractional integration of the $\bar{H}$-function. Matematiche $67(2), 107-118$ (2012)

17. Agarwal, P: Fractional integration of the product of two multivariables $\mathrm{H}$-function and a general class of polynomials. In: Anastassiou, GA, Duman, O (eds.) Advances in Applied Mathematics and Approximation Theory, vol. 41, pp. 359-374. Springer, New York (2013)

18. Baleanu, D, Agarwal, P: On generalized fractional integral operators and the generalized Gauss hypergeometric functions. Abstr. Appl. Anal. 2014, Article ID 630840 (2014). doi:10.1155/2014/630840

19. Choi, J, Agarwal, P: Certain integral transform and fractional integral formulas for the generalized Gauss hypergeometric functions. Abstr. Appl. Anal. 2014, Article ID 735946 (2014). doi:10.1155/2014/735946

20. Srivastava, HM, Agarwal, P: Certain fractional integral operators and the generalized incomplete hypergeometric functions. Appl. Appl. Math. 8(2), 333-345 (2013)

21. Saxena, RK: On the $H$-function of $n$ variables. Kyungpook Math. J. 17, 221-226 (1977)

22. Srivastava, HM, Panda, R: Expansion theorems for the $H$-function of several complex variables. J. Reine Angew. Math. 288, 129-145 (1976)

23. Saigo, M: On generalized fractional calculus operators. In: Recent Advances in Applied Mathematics (Proc. Internat. Workshop held at Kuwait Univ.), Kuwait Univ., Kuwait, pp. 441-450 (1996)

24. Kiryakova, V: On two Saigo's fractional integral operators in the class of univalent functions. Fract. Calc. Appl. Anal. 9(2), 160-176 (2006)

25. McBride, AC: Fractional Calculus and Integral Transforms of Generalized Functions. Research Notes in Math., vol. 31. Pitman, London (1979)

26. Olver, FWJ, Lozier, DW, Boisvert, RF, Clark, CW (eds.): NIST Handbook of Mathematical Functions. Cambridge University Press, Cambridge (2010)

27. Saigo, M: A remark on integral operators involving the Gauss hypergeometric functions. Math. Rep. Coll. Gen. Educ. Kyushu Univ. 11, 135-143 (1978)

28. Srivastava, HM, Panda, R: Some bilateral generating functions for a class of generalized hypergeometric polynomials. J. Reine Angew. Math. 283/284, 265-274 (1976)

29. Srivastava, HM, Panda, R: Certain multidimensional integral transformations. I. Ned. Akad. Wet. Proc., Ser. A, Indag. Math. 40, 118-131 (1978)

30. Srivastava, HM, Panda, R: Certain multidimensional integral transformations. II. Ned. Akad. Wet. Proc., Ser. A, Indag. Math. 40, 132-144 (1978) 
31. Srivastava, HM, Gupta, KC, Goyal, SP: The H-Functions of One and Two Variables with Applications. South Asian Publishers, New Delhi (1982)

32. Srivastava, HM, Panda, R: Some expansion theorems and generating relations for the $H$-functions of several complex variables. II. Comment. Math. Univ. St. Pauli 25, 169-197 (1976)

33. Mittal, PK, Gupta, KC: An integral involving generalized function of two variables. Proc. Indian Acad. Sci., Sect. A 75, $117-123(1972)$

34. Munot, PC, Kalla, SL: On an extension of generalized function of two variables. Rev. Univ. Nac. Tucumán Ser. A 21, 67-84 (1971)

35. Verma, RU: On the H-function of two variables - II. An. Ştiinţ. Univ. 'Al.I. Cuza' laşi, Mat. 17, 103-109 (1971)

36. Hai, NT, Yakubovich, SB: The Double Mellin-Barnes Type Integrals and Their Applications to Convolution Theory. World Scientific, Singapore (1992)

37. Srivastava, HM, Panda, R: Expansion theorems for the H-function of several complex variables. J. Reine Angew. Math. 288, 129-145 (1976)

38. Saxena, RK: On a generalized function of $n$ variables. Kyungpook Math. J. 14, 255-259 (1974)

39. Khadia, SS, Goyal, AN: On the generalized function of $n$ variables - II. Vijnana Parishad Anusandhan Patrika 18, 359-366 (1975)

40. Khadia, SS, Goyal, AN: On the generalized function of $n$-variables. Vijnana Parishad Anusandhan Patrika 13, 191-201 (1970)

41. Saigo, M, Saxena, RK: Unified fractional integral formulas for the multivariable $H$-function. J. Fract. Calc. 15, 91-107 (1999)

42. Saigo, M, Saxena, RK: Unified fractional integral formulas for the multivariable H-function - II. J. Fract. Calc. 16, 99-110 (1999)

43. Saigo, M, Saxena, RK: Unified fractional integral formulas for the multivariable H-function - III. J. Fract. Calc. 20, 45-68 (2001)

44. Srivastava, HM, Hussain, MA: Fractional integration of the H-function of several variables. Comput. Math. Appl. 30, 73-85 (1995)

45. Saigo, M, Saxena, RK, Ram, J: Fractional integration of the product of Appell function $F_{3}$ and multivariable $\mathrm{H}$-function. J. Fract. Calc. 27, 31-42 (2005)

46. Srivastava, HM, Garg, M: Some integrals involving a general class of polynomials and multivariable $H$-function. Rev. Roum. Phys. 32, 685-692 (1987)

47. Agarwal, P: Further results on fractional calculus of Saigo operator. Appl. Appl. Math. 7(2), 585-594 (2012)

48. Srivastava, HM, Singh, NP: The integration of certain product of the multivariable $H$-function with a general class of polynomials. Rend. Circ. Mat. Palermo 32, 157-187 (1983)

49. Kilbas, AA, Saigo, M: H-Transforms: Theory and Application. Chapman \& Hall/CRC, Boca Raton (2004)

50. Kilbas, AA, Sebastian, N: Generalized fractional integration of Bessel function of first kind. Integral Transforms Spec. Funct. 19(12), 869-883 (2008)

51. Prabhakar, TR: A singular integral equation with a generalized Mittag-Leffler function in the kernel. Yokohama Math. J. 19, 7-15 (1971)

52. Gorenflo, R, Kilbas, A, Mainardi, F, Rogosin, S: Mittag-Leffler Function, Related Topics and Applications. Springer, Berlin (2014)

53. Saxena, RK, Ram, J, Suthar, DL: Fractional calculus of generalized Mittag-Leffler functions. J. Indian Acad. Math. 31(1), 165-172 (2009)

54. Kilbas, AA: Fractional calculus of the generalized Wright function. Fract. Calc. Appl. Anal. 8(2), 113-126 (2005)

55. Srivastava, HM, Saxena, RK, Ram, J: Some multidimensional fractional integral operations involving a general class of polynomials. J. Math. Anal. 193, 373-389 (1995)

\section{Submit your manuscript to a SpringerOpen ${ }^{\circ}$ journal and benefit from:}

- Convenient online submission

Rigorous peer review

- Immediate publication on acceptance

- Open access: articles freely available online

- High visibility within the field

- Retaining the copyright to your article 